\title{
On intercalated Fibonacci sequences
}

\author{
Krassimir T. Atanassov ${ }^{1}$ and Anthony G. Shannon ${ }^{2}$ \\ ${ }^{1}$ Department of Bioinformatics and Mathematical Modelling \\ IBPhBME, Bulgarian Academy of Sciences \\ Acad. G. Bonchev Str., Bl. 105, Sofia--1113, Bulgaria \\ e-mail: krat@bas.bg \\ ${ }^{2}$ Warrane College, The University of New South Wales \\ 356 Anzac Parade, Kensington, NSW 2033, Australia \\ e-mail: t. shannon@warrane. unsw. edu.au; tshannon38@gmail.com
}

Abstract: We construct three intercalated sequences and develop their essential properties which are generalizations of the three basic Fibonacci sequences. They are extensions of pulsated sequences described at previous Fibonacci conferences. We relate these sequences to the sequence $\left\{y_{n}\right\}_{n} \geq 0=\{0,1,4,15,56, \ldots\}$.

Keywords: Fibonacci-type sequences, Recurrence relations, Induction, Horadam sequences. 2010 Mathematics Subject Classification: 11B37, $11 \mathrm{~B} 39$.

\section{Introduction}

In a series of joint papers, the authors introduced more than ten different Fibonacci-type sequences (see, $[1-20,24]$ ). In the present paper, we introduce a new Fibonacci type sequence which is related to the pulsated Fibonacci sequence $[7,12,19]$.

We begin with the construction of the following three sequences in Table 1.

These sequences are obtained from the following initial terms and recurrence relations

$$
\begin{gathered}
\alpha_{0}=a, \beta_{0}=b, \gamma_{0}=c \\
\alpha_{n+1}=\beta_{n}+\gamma_{n}+\alpha_{n}, \beta_{n+1}=\alpha_{n+1}+\gamma_{n} ; \gamma_{n+1}=\alpha_{n+1}+\beta_{n}
\end{gathered}
$$

for each natural number $n \geq 0$. It is the purpose of this note to show how they are interrelated. 


\begin{tabular}{|c|c|c|c|}
\hline $\boldsymbol{n}$ & $\boldsymbol{\beta}_{\boldsymbol{n}}$ & $\boldsymbol{\alpha}_{\boldsymbol{n}}$ & $\boldsymbol{\gamma}_{\boldsymbol{n}}$ \\
\hline 0 & & $a$ & $c$ \\
\hline & $b$ & $a+b+c$ & $a+2 b+c$ \\
\hline 1 & $a+b+2 c$ & & $4 a+5 b+6 c$ \\
\hline & & $3 a+4 b+4 c$ & \\
\hline 2 & $4 a+6 b+5 c$ & & $15 a+21 b+20 c$ \\
\hline & & $11 a+15 b+15 c$ & \\
\hline 3 & $15 a+20 b+21 c$ & & $56 a+76 b+77 c$ \\
\hline & & $41 a+56 b+56 c$ & $\ldots$ \\
\hline 4 & $56 a+77 b+76 c$ & $\ldots$ & \\
\hline & $\ldots$ & $\ldots$ & \\
\hline$\ldots$ & & & \\
\hline
\end{tabular}

Table 1. $\left\{\alpha_{n}\right\},\left\{\beta_{n}\right\},\left\{\gamma_{n}\right\}$

\section{Preliminary results}

Let us define

$$
\begin{aligned}
\alpha_{n} & =x_{n} a+y_{n} b+y_{n} c \\
\beta_{n} & =p_{n} a+q_{n} b+r_{n} c \\
\gamma_{n} & =p_{n} a+r_{n} b+q_{n} c
\end{aligned}
$$

We see and can prove, for example, by induction, that for each natural number $n \geq 0$ :

$$
\begin{aligned}
x_{n} & =y_{n}-y_{n-1} \\
p_{n} & =y_{n} \\
q_{n} & =r_{n}+(-1)^{n} \\
r_{n} & =q_{n}+(-1)^{n-1} \\
q_{n}+r_{n} & =y_{n+1}-y_{n} .
\end{aligned}
$$

Therefore,

$$
\begin{aligned}
q_{n} & =\frac{1}{2}\left(y_{n+1}-y_{n}+(-1)^{n}\right), \\
r_{n} & =\frac{1}{2}\left(y_{n+1}-y_{n}+(-1)^{n+1}\right) .
\end{aligned}
$$

Hence, all coefficients can be represented by coefficients $y_{n}$ for each natural number $n \geq 0$, and we see that the sequence $\left\{y_{n}\right\}_{n} \geq 0=\{0,1,4,15,56, \ldots\}$ has a common member that is obtained from the equation

$$
y^{2}-4 y+1=0
$$

which has squares $2+\sqrt{3}$ and $2-\sqrt{3}$. Hence,

$$
y_{n}=\frac{1}{2 \sqrt{3}}\left((2+\sqrt{3})^{n}-(2-\sqrt{3})^{n}\right)
$$

and

$$
y_{n+2}=4 y_{n+1}-y_{n} .
$$


Therefore, we can represent all other coefficients in terms of the coefficients in $y_{n}$ as follows

$$
\begin{aligned}
x_{n} & =y_{n}-y_{n-1} \\
& =\frac{1}{2 \sqrt{3}}\left((2+\sqrt{3})^{n}-(2-\sqrt{3})^{n}\right)-\frac{1}{2 \sqrt{3}}\left((2+\sqrt{3})^{n-1}-(2-\sqrt{3})^{n-1}\right) \\
& =\frac{1}{2 \sqrt{3}}\left((2+\sqrt{3})^{n-1}(1+\sqrt{3})-(2-\sqrt{3})^{n-1}(1-\sqrt{3})\right) \\
& =\frac{1}{2 \sqrt{3}}\left((2+\sqrt{3})^{n-1}-(2-\sqrt{3})^{n-1}+\sqrt{3}\left((2+\sqrt{3})^{n-1}+(2-\sqrt{3})^{n-1}\right)\right) \\
& =\frac{1}{2 \sqrt{3}}\left((2+\sqrt{3})^{n-1}-(2-\sqrt{3})^{n-1}+\frac{1}{2}\left((2+\sqrt{3})^{n-1}+(2-\sqrt{3})^{n-1}\right)\right) ;
\end{aligned}
$$

and

$$
\begin{aligned}
& p_{n}=\frac{1}{2+\sqrt{3}}\left((2+\sqrt{3})^{n}-(2-\sqrt{3})^{n}\right) ; \\
& \left.q_{n}=\frac{1}{2}\left(\frac{1}{2 \sqrt{3}}\left((2+\sqrt{3})^{n-1}-(2-\sqrt{3})^{n-1}\right)+\frac{1}{2}\left((2+\sqrt{3})^{n-1}+(2-\sqrt{3})^{n-1}\right)\right)+(-1)^{n}\right) ; \\
& \left.r_{n}=\frac{1}{2}\left(\frac{1}{2 \sqrt{3}}\left((2+\sqrt{3})^{n-1}-(2-\sqrt{3})^{n-1}\right)+\frac{1}{2}\left((2+\sqrt{3})^{n-1}+(2-\sqrt{3})^{n-1}\right)\right)+(-1)^{n+1}\right) .
\end{aligned}
$$

Finally, we can formulate and prove by induction the following result.

\section{Main result}

Theorem 1. For each natural number $n \geq 1$ the general terms ( $n$-th members) of the three sequences $\left\{\alpha_{n}\right\}_{n \geq 0},\left\{\beta_{n}\right\}_{n \geq 0}$ and $\left\{\gamma_{n}\right\}_{n \geq 0}$ are respectively

$$
\begin{aligned}
\alpha_{n}= & \left(\frac{1}{2 \sqrt{3}}\left((2+\sqrt{3})^{n-1}-(2-\sqrt{3})^{n-1}\right)+\frac{1}{2}\left((2+\sqrt{3})^{n-1}+(2-\sqrt{3})^{n-1}\right)\right) a \\
& +\frac{1}{2 \sqrt{3}}\left((2+\sqrt{3})^{n}-(2-\sqrt{3})^{n}\right)(b+c) ; \\
\beta_{n}= & \frac{1}{2 \sqrt{3}}\left((2+\sqrt{3})^{n}-(2-\sqrt{3})^{n}\right) a+\frac{1}{2}\left(\frac{1}{2 \sqrt{3}}\left((2+\sqrt{3})^{n-1}-(2-\sqrt{3})^{n-1}\right)\right. \\
& \left.+\frac{1}{2}\left((2+\sqrt{3})^{n-1}+(2-\sqrt{3})^{n-1}\right)+(-1)^{n}\right) b+\frac{1}{2}\left(\frac{1}{2 \sqrt{3}}\left((2+\sqrt{3})^{n-1}-(2-\sqrt{3})^{n-1}\right)\right. \\
& \left.+\frac{1}{2}\left((2+\sqrt{3})^{n-1}+(2-\sqrt{3})^{n-1}\right)+(-1)^{n+1}\right) c ; \\
\gamma_{n}= & \frac{1}{2 \sqrt{3}}\left((2+\sqrt{3})^{n}-(2-\sqrt{3})^{n}\right) a+\frac{1}{2}\left(\frac{1}{2 \sqrt{3}}\left((2+\sqrt{3})^{n-1}-(2-\sqrt{3})^{n-1}\right)\right. \\
& \left.+\frac{1}{2}\left((2+\sqrt{3})^{n-1}+(2-\sqrt{3})^{n-1}\right)+(-1)^{n+1}\right) b+\frac{1}{2}\left(\frac{1}{2 \sqrt{3}}\left((2+\sqrt{3})^{n-1}-(2-\sqrt{3})^{n-1}\right)\right. \\
& \left.\left.+\frac{1}{2}\left((2+\sqrt{3})^{n-1}+(2-\sqrt{3})^{n-1}\right)+(-1)^{n}\right)\right) c .
\end{aligned}
$$


Proof. When $n=1,2$, the validity of the assertion is seen from the definition of the sequences. Let assume that the assertion is valid for some $n \geq 2$. Now, we shall check its validity for $n+1$, using the shorter form of the representations of $\alpha_{n}, \beta_{n}$ and $\gamma_{n}$ :

$$
\begin{aligned}
\alpha_{n+1} & =\beta_{n}+\gamma_{n}+\alpha_{n} \\
& =p_{n} a+q_{n} b+r_{n} c+p_{n} a+r_{n} b+q_{n} c+x_{n} a+y_{n} b+y_{n} c \\
& =\left(2 p_{n}+x_{n}\right) a+\left(q_{n}+r_{n}+y_{n}\right) b+\left(q_{n}+r_{n}+y_{n}\right) c \\
& =\left(2 y_{n}+y_{n}-y_{n-1}\right) a+\left(y_{n+1}-y_{n}+y_{n}\right) b+\left(y_{n+1}-y_{n}+y_{n}\right) c \\
& =\left(3 y_{n}-y_{n-1}\right) a+y_{n+1} b+y_{n+1} c \\
& \quad \quad\left[\text { from } x_{n+1}=y_{n+1}-y_{n}=4 y_{n}-y_{n-1}-y_{n}=3 y_{n}-y_{n-1}\right] \\
\beta_{n+1}= & y_{n+1} a+y_{n+1} b+y_{n+1} c ; \quad \gamma_{n} \quad \quad\left[\text { from } y_{n+2}=4 y_{n+1}-y_{n}\right] \\
= & x_{n+1} a+y_{n+1} b+y_{n+1} c+p_{n} a+r_{n} b+q_{n} c \\
= & \left(x_{n+1}+p_{n}\right) a+\left(y_{n+1}+r_{n}\right) b+\left(y_{n+1}+q_{n}\right) c \\
= & \left(p_{n+1}-p_{n}+p_{n}\right) a+\left(y_{n+1}+r_{n}\right) b+\left(y_{n+1}+q_{n}\right) c \\
= & p_{n+1} a+\left(y_{n+1}+\frac{1}{2}\left(y_{n+1}-y_{n}+(-1)^{n+1}\right)\right) b+\left(y_{n+1}+\frac{1}{2}\left(y_{n+1}-y_{n}+(-1)^{n}\right)\right) c \\
= & p_{n+1} a+\frac{1}{2}\left(3 y_{n+1}-y_{n}+(-1)^{n+1}\right) b+\frac{1}{2}\left(3 y_{n+1}-y_{n}+(-1)^{n}\right) c \\
= & p_{n+1} a+\frac{1}{2}\left(4 y_{n+1}-y_{n+1}-y_{n}+(-1)^{n+1}\right) b+\frac{1}{2}\left(4 y_{n+1}-y_{n+1}-y_{n}\right) c \\
= & p_{n+1} a+\frac{1}{2}\left(y_{n+2}-y_{n+1}+(-1)^{n+1}\right) b+\frac{1}{2}\left(y_{n+2}-y_{n+1}+(-1)^{n}\right) c \\
& p_{n+1} a+q_{n+1} b+r_{n+1} c .
\end{aligned}
$$

The check for $\gamma_{n+1}$ is identical to that for $\beta_{n+1}$.

This completes the proof.

\section{Conclusion}

We can see some of the foregoing with a simplistic numerical example, in which $a=1, b=2$ and $c=3$, as set out in Table 2. The examples are trivial, but their interdependence can be clearly seen as part of their 'basic' nature, and some of those in [25; A001075, A001835] have a rich history. $\left\{x_{n}\right\}$ satisfies the same second order linear homogeneous recurrence relation as $\left\{y_{n}\right\}$ but with different initial conditions as in Horadam sequences [22].

The $\left\{y_{n}\right\}$ sequence is shown in [25] to have applications in other parts of number theory (such as prime-free sequences), geometry, combinatorics, special functions, numerical analysis and Hessenberg matrices. It is related to many other classes of sequences [21, 23]. 


\begin{tabular}{|c|c|c|c|c|c|c|c|c|}
\hline $\boldsymbol{n}$ & $\boldsymbol{\beta}_{\boldsymbol{n}}$ & $\boldsymbol{\alpha}_{\boldsymbol{n}}$ & $\boldsymbol{\gamma}_{\boldsymbol{n}}$ & $\boldsymbol{y}_{\boldsymbol{n}}$ & $\boldsymbol{x}_{\boldsymbol{n}}$ & $\boldsymbol{x}_{\boldsymbol{n}}+\boldsymbol{y}_{\boldsymbol{n}}$ & $\boldsymbol{\gamma}_{\boldsymbol{n}}-\boldsymbol{\alpha}_{\boldsymbol{n}}$ & $\boldsymbol{\gamma}_{\boldsymbol{n}}-\boldsymbol{\beta}_{\boldsymbol{n}}$ \\
\hline 0 & 2 & 1 & 3 & 0 & 1 & 1 & 2 & 1 \\
\hline 1 & 9 & 6 & 8 & 1 & 1 & 2 & 2 & -1 \\
\hline 2 & 31 & 23 & 32 & 4 & 3 & 7 & 9 & 1 \\
\hline 3 & 118 & 86 & 117 & 15 & 11 & 26 & 31 & -1 \\
\hline 4 & 438 & 321 & 439 & 56 & 41 & 97 & 118 & 1 \\
\hline
\end{tabular}

Table 2. Numerical examples of sequences, $n=0,1,2,3,4$

\section{References}

[1] Atanassov, K. (1986). On a second new generalization of the Fibonacci sequence. The Fibonacci Quarterly, 24 (4), 362-365.

[2] Atanassov, K. (1989). Remark on variants of Fibonacci squares. Bulletin of Number Theory and Related Topics, XIII, 25-27.

[3] Atanassov, K. (1989). A remark on a Fibonacci plane. Bulletin of Number Theory and Related Topics, XIII, 69-71.

[4] Atanassov, K. (1989). On a generalization of the Fibonacci sequence in the case of three sequences. The Fibonacci Quarterly, 27 (1), 7-10.

[5] Atanassov, K. (1995). Remark on a new direction for a generalization of the Fibonacci sequence, The Fibonacci Quarterly, 33 (3), 249-250.

[6] Atanassov, K. (2006). A new direction of Fibonacci sequence modification. Notes on Number Theory and Discrete Mathematics, 12 (1), 25-32.

[7] Atanassov, K. (2014). n-Pulsated Fibonacci sequence. Notes on Number Theory and Discrete Mathematics, 20 (1), 32-35.

[8] Atanassov, K. (2017). On two new two-dimensional extensions of the Fibonacci sequence. Notes on Number Theory and Discrete Mathematics, 23 (3), 115-122.

[9] Atanassov, K. (2018). On two new combined 3-Fibonacci sequences. Notes on Number Theory and Discrete Mathematics, 24 (2), 90-93.

[10] Atanassov, K., Atanassova, L., \& Sasselov, D. (1985). A new perspective to the generalization of the Fibonacci sequence, The Fibonacci Quarterly, 23 (1), 21-28.

[11] Atanassov K., Atanassova, V., Shannon, A., \& Turner, J. (2002). New Visual Perspectives on Fibonacci Numbers. World Scientific, New Jersey.

[12] Atanassov, K., Deford, D. R., \& Shannon, A. G. Atanassov, K. (2014). Pulsated Fibonacci recurrences. Proceedings of the Sixteenth International Conference on Fibonacci Numbers and Their Applications (P. Anderson, C. Ballot, W. Webb, Eds.), Rochester, New York, July 20-27, 2014, 22-27.

[13] Atanassov, K., \& Shannon A. G. (1999). Fibonacci planes and spaces. In Applications of Fibonacci Numbers (F. Howard, Ed.), Vol. 8, Dordrecht, Kluwer, 43-46. 
[14] Atanassov, K., \& Shannon A. G. (2002). New variant of a Fibonacci plane. Notes on Number Theory and Discrete Mathematics, 8 (3), 112-115.

[15] Atanassov, K., \& Shannon A. G. (2005). Digit sum bases for Fibonacci and related numbers. Notes on Number Theory and Discrete Mathematics, 11 (3), 25-32.

[16] Atanassov, K., \& Shannon A. G. (2008). A Fibonacci cylinder, Notes on Number Theory and Discrete Mathematics, 14 (4), 4-9.

[17] Atanassov, K., \& Shannon A. G. (2010). A short remark on Fibonacci-type sequences, Mobius strips and the $\psi$-function. International Journal of Mathematical Education in Science and Technology, 41 (8), 1125-1127.

[18] Atanassov, K., \& Shannon A. G. (2011). The digital root function for Fibonacci-type sequences. Advanced Studies in Contemporary Mathematics, 21 (3), 251-254.

[19] Atanassov, K., \& Shannon A. G. (2016). Combined 3-Fibonacci sequences from a new type. Notes on Number Theory and Discrete Mathematics, 22 (3), 5-8.

[20] Atanassova, V., Shannon, A., \& Atanassov, K. (2003). Sets of extensions of the Fibonacci sequence. Comptes Rendus de l'Academie bulgare des Sciences, 56 (9), 9-12.

[21] Everest, G., Van der Poorten, A., Shparlinski, I., \& Ward, T. (2003). Recurrence Sequences (Mathematical Surveys and Monographs; Vol. 104). Providence: RI: American Mathematical Society, p. 163.

[22] Horadam, A. F. (1967). Special properties of the sequence $\left\{w_{n}(a, b ; p, q)\right\}$. The Fibonacci Quarterly, 5 (5), 424-434.

[23] Janjić, M., \& Petković, B. (2014). A Counting Function Generalizing Binomial Coefficients and Some Other Classes of Integers. Journal of Integer Sequences, 17, Article ID 14.3.5.

[24] Shannon, A., \& Atanassov, K. (2002). Introduction to the difference calculus through the Fibonacci numbers. International Journal of Mathematical Education in Science and Technology, 33 (3), 456-465.

[25] Sloane, N. J. A., \& Plouffe, S. (1995). The Encyclopedia of Integer Sequences, San Diego, CA: Academic Press, A001353. 